\title{
SEMI-ACTIVE LINEAR VACUUM PACKED PARTICLES DAMPER
}

\author{
Robert Zalewski, PaweŁ Chodkiewicz \\ Warsaw University of Technology, Institute of Machine Design Fundamentals, Warsaw, Poland \\ e-mail: robertzalewski@wp.pl; pawel@chodkiewicz.com.pl
}

\begin{abstract}
In this paper, the authors focus on the proposition of an innovative semi-active linear damper prototype working on the basis of granular materials. Vacuum Packed Particles (VPP) belong to the class of materials whose mechanical (rheological, dissipative) properties may be quickly changed by applying a partial vacuum inside the system. The concept of an innovative linear damper based on VPP is presented in the paper. Typical experimental results are presented to reveal changeable damping characteristics of the device. Additionally, the mathematical model is proposed to capture extraordinary features of the investigated damper.
\end{abstract}

Keywords: Vacuum Packed Particles, modeling, underpressure, experiments

\section{Introduction}

Vacuum Packed Particles (VPP) are a class of "smart structures" whose physical properties may be rapidly changed by pulling out the pressure from the system and generating the so called underpressure. This change is in proportion to the magnitude of the internal partial vacuum generated and is quickly reversible. VPP, from the macroscopic point of view, are viscoplastic solid bodies and can be modeled by various constitutive models (Zalewski and Pyrz, 2013). The VPP structure can be also compared to magnetorheological materials and, consequently, modeled by typical rheological models developed for MR fluids. Typical, well commercialized engineering applications of VPP are universal robot grippers (Brown et al., 2010), flexible endoscopes (Loeve et al., 2010), "smart layers" in sandwich beam structures (Bajkowski et al., 2015) or vacuum mattresses (Luscombe and Williams, 2003).

The discussed granular structures are conglomerates that consist of loose granular materials placed in a soft and hermetic envelope. When exposed to a partial vacuum, the so called "jamming mechanism" occurs and loose particles interact to form a solid-like structure that resists various types of deformations or flow (Cates et al., 1998). This change in the structure appears as a dramatic increase in apparent viscosity, and the "plastic" structure develops characteristics of a semisolid state (Majmudar et al., 2007). The magnitude of this transformation is controlled by the value of the partial vacuum and is immediately reversed upon removing the underpressure.

Noting the apparent similarities of the considered VPP and magnetorheological (MR) fluids (Makowski and Knap, 2014), the authors propose an "innovative" semi-active linear VPP damper prototype. Taking advantage of the previous, fundamental research on VPP, in this paper the authors propose an original engineering application of the previously mentioned granular conglomerates. In the modeling Section, a mathematical model including damage functions is proposed. Typical laboratory tests results are presented in the experimental Section. The impact of underpressure on recorded dissipative characteristics is introduced and discussed. The model has been calibrated using an Evolutionary Algorithm. 


\section{Model of the system}

In our experiment we consider a system that consists of a spring that is attached to a rigid support at the top and has a mass attached to it at the bottom end (Fig. 1). The spring encloses a flexible and hermetically sealed sleeve that is full with a granular material, which acts as the damper.

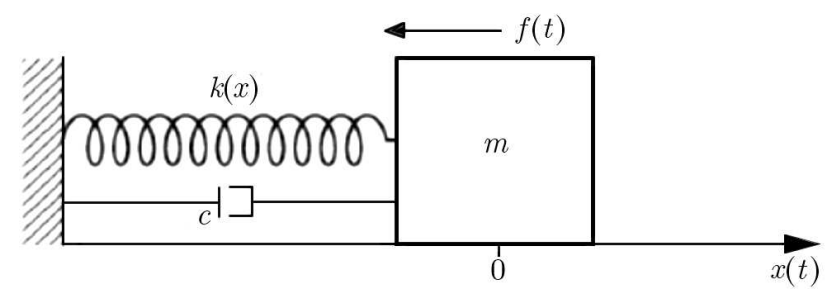

Fig. 1. Model of the nonlinear mass-spring-damper system

To capture real behavior of the proposed VPP damper prototype, the following mathematical model is proposed

$$
\begin{aligned}
& \dot{v}+c \zeta v+k x+\mu g \operatorname{sgn}(v)=f \\
& D(t)=d_{s} \int_{0}^{t}|v(s)| d s \\
& \dot{\zeta}=-d_{f}\left(|x|-\lambda_{f}\right)_{+}-D \\
& x(0)=x_{0} \quad v(0)=v_{0} \quad \zeta(0)=\zeta_{0}
\end{aligned}
$$

where $m, k, c$ are positive material coefficients, $\mu$ is the coefficient of friction and $g$ is the gravitational acceleration. The introduced $D$ function with the rate $d_{s}$ is related to gradual wear of single grains caused by the "intergranular" friction phenomenon. The global damage function $\zeta$ also consists of the part related to rearrangement of the grains along the total path traveled. The damping damage coefficients $d_{f}$ and $d_{s}$ are based on experiments, and we assume that they depend on the underpressure. $\lambda_{f}$ is the critical amplitude below which the granular material does not change its internal arrangement.

The problem is rewritten as a system of three first order deferential equations with appropriate initial conditions. A forward-type Euler algorithm (2.2) has been used to solve the system $(2.1)$

$$
\begin{aligned}
& v_{n+1}=v_{n}-\frac{c v_{n}-k x_{n}+\mu g \operatorname{sgn}\left(v_{n}\right)+f_{n+1}}{m} h \\
& x_{n+1}=x_{n}+v_{n+1} h \\
& D_{n+1}=D_{n}+d_{s}\left|v_{n+1}\right| h \\
& \zeta_{n+1}=\zeta_{n}-\left[d_{f}\left(\left|x_{n+1}\right|-\lambda_{f}\right)_{+}-D_{n+1}\right] h
\end{aligned}
$$

\section{Experiments}

The structural scheme of the device is depicted in Fig. 2. It consists of two rigid discs $(3,5)$, coupled by the main spring (2). The heart of the device is a granular core (4). It is formed of a cylindrical envelope filled by loose plastomer grains (also small cylinders). Thanks to the special valve mounted in a handle (1), it is possible to connect the core to a vacuum pump and generate the appropriate value of a partial vacuum inside the system. 


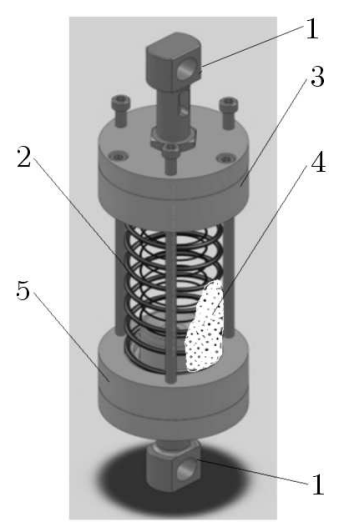

Fig. 2. Scheme of the investigated damper; 1 - handles, 2 - spring, 3 - upper disc, 4 - granular core, 5 - lower disc

The VPP damper prototype has been investigated on a specially designed laboratory stand (Fig. 3). A kinematical sine excitation rule with various frequencies was considered in the laboratory tests. Different underpressure values from the range 0.01 to $0.09 \mathrm{MPa}$ were taken into considerations. Typical experimental results are depicted in Fig. 4.

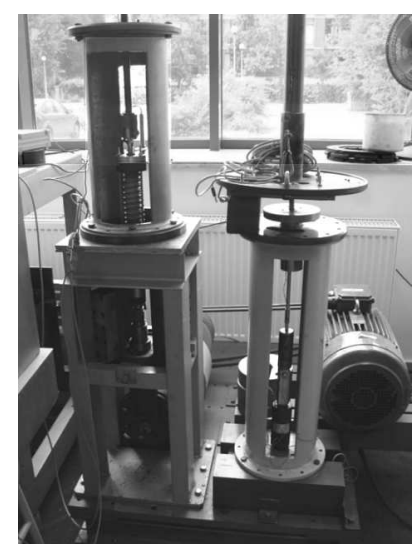

Fig. 3. Laboratory stand for investigations of VPP dampers

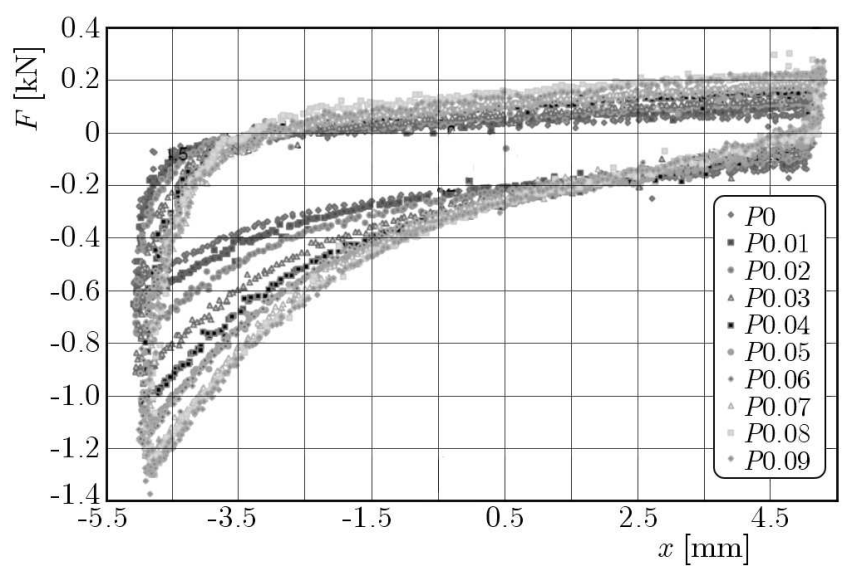

Fig. 4. Damping characteristics of the VPP damper for various values of underpressure and the excitation frequency $f=1.4 \mathrm{~Hz}$

Analyzing the experimental results presented in Fig. 4, it can be observed that the underpressure parameter has a great impact on the recorded energy dissipation loops. A higher 
underpressure value results in increased damping properties of the VPP damper prototype. This phenomenon shows that changing the value of partial vacuum inside the system enables one to control the global properties of the discussed devices. In the authors opinion, it confirms that investigated devices can be placed among the family of "smart dampers" next to MR or ER devices.

The recorded data also reveal a nonsymmetrical response of the damper, which complicates the mathematical description of the damper. The results of laboratory tests, in the next stage of research, are the base for the mathematical model calibration process.

\section{Model calibration}

The model has been calibrated for two various values of the underpressure. We used the Evolutionary Algorithm (EA) optimization method to find 6 parameters of the model presented in Section 2. The EA developed in the Mathematica software applies a population of 40 individuals and simulated the evolution process for 200 generations. Each iterative step includes three stages: selection, mutation and crossover. Crossover and mutation operators are applied randomly with $50 \%$ probability. The following fitness function is taken into consideration

$$
E_{r}=\frac{1}{n} \sum_{i=1}^{n} \frac{\left|F_{e x p}^{i}-F^{i}\right|}{\left|F_{e x p}^{i}\right|} \rightarrow \min
$$

where $F_{\exp }$ is a temporary experimental force value, $F$ - numerical force value, $n$ - total number of discrete points.
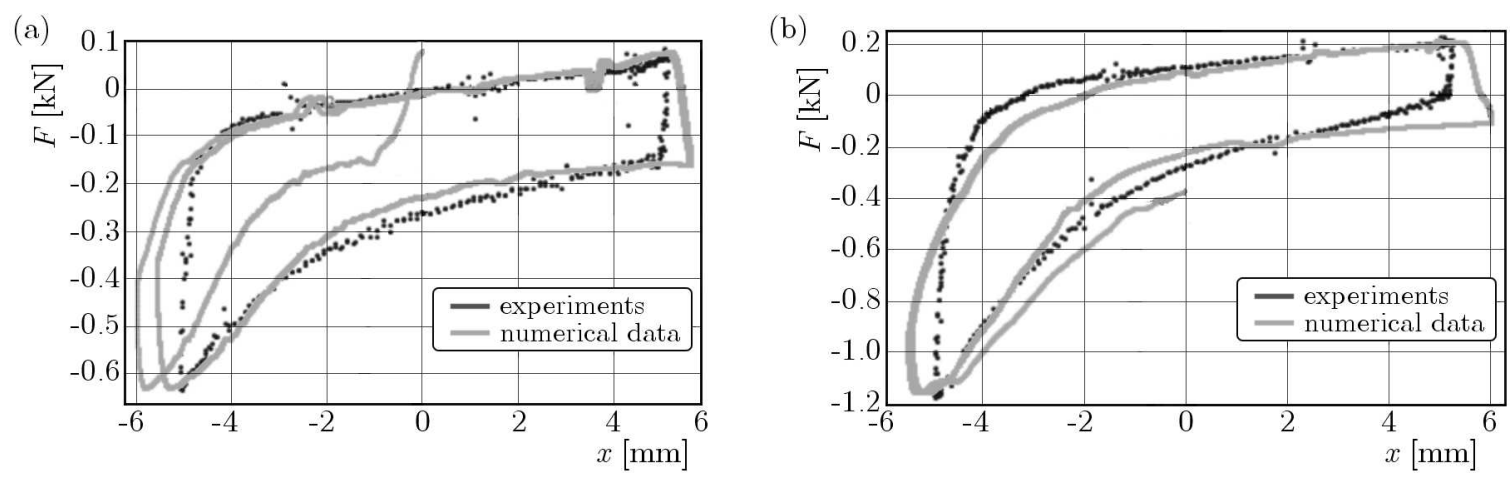

Fig. 5. Verification of the model calibration processes for (a) $P=0.01 \mathrm{MPa}$; (b) $P=0.07 \mathrm{MPa}$

The old population is replaced by new individuals for which new fitness values are calculated. The best results obtained after 200 iterations are presented in Table 1. The numerical simulation results carried out for the identified model parameters have been verified with direct experimental data (Fig. 5). In the initial step of numerical investigations, damage functions $(2.1)_{2,3}$ were turned off during the calibration process. As the properties of the VPP damper are influenced by the underpressure value and the direction of velocity (Fig. 4), we assumed that all investigated parameters can be described as

$$
\begin{aligned}
& c(P, \operatorname{sgn}(v))=\left\{\begin{array}{ll}
c_{0} & \text { for } \quad v \geqslant 0 \\
c_{1} & \text { for } \quad v<0
\end{array} \quad k(P, \operatorname{sgn}(v))= \begin{cases}k_{0} & \text { for } \quad v \geqslant 0 \\
k_{1} & \text { for } \quad v<0\end{cases} \right. \\
& n(P, \operatorname{sgn}(v))=\left\{\begin{array}{lll}
n_{0} & \text { for } \quad v \geqslant 0 \\
-n_{1} & \text { for } \quad v<0
\end{array}\right.
\end{aligned}
$$


Finally, the estimated values of the model parameters for two selected underpressures $(0.01$ and $0.07 \mathrm{MPa}$ ) are presented in Table 1 . For the simplicity of calculations it has been assumed that $n=\mu g$.

Table 1. Model parameters for various underpressure values

\begin{tabular}{|c|c|c|c|c|c|c|}
\hline$P[\mathrm{MPa}]$ & $c_{0}[\mathrm{~kg} / \mathrm{s}]$ & $k_{0}[\mathrm{kN} / \mathrm{mm}]$ & $c_{1}[\mathrm{~kg} / \mathrm{s}]$ & $k_{1}[\mathrm{kN} / \mathrm{mm}]$ & $n_{0}[\mathrm{kN}]$ & $n_{1}[\mathrm{kN}]$ \\
\hline \hline 0.01 & 34.14 & 42.22 & 50.57 & 145.51 & 0.40 & 0.44 \\
\hline 0.07 & 72.53 & 117.96 & 51.10 & 297.13 & 0.82 & 0.69 \\
\hline
\end{tabular}

\section{Conclusions}

In the paper, an innovative semi-active damper prototype, based on Vacuum Packed Particles is proposed and investigated. The experimental results confirmed the possibility of controlling the dissipative properties of the device by changing the value of partial vacuum. Higher underpressure provides intensification of grains compaction and results in increasing the damping properties of the device.

The proposed mathematical model assumes two types of damage functions. Most important damage mechanisms, encountered during experimental research, are related to the ongoing wear of single grains material and large, exceeding the assumed range, rearrangements of the granular system.

The model has been calibrated basing on the obtained experimental results using the EA strategy. Verification of the numerical and laboratory tests results revealed quite a good correctness of the proposed model (global error less than 7\%).

The previous experimental research did not include destructive tests of the investigated VPP prototype. To identify the damage functions, a multi-cycle loading of the testing device has to be applied. Moreover, the nonlinear underpressure functions have to be identified and introduced to the proposed model.

Future design works should be focused on developing semi-active granular devices with optional, not necessarily nonsymmetrical damping characteristics.

In the authors' opinion, semi-active linear VPP dampers may in a near future replace much more expensive and complex magnetorheological or electrorheological devices. More than 500 times cheaper and uncomplicated VPP dampers seem to be competitive to already well commercialized and popular "intelligent" dampers.

\section{References}

1. Bajkowski J.M., Dyniewicz B., Bajer C.I., 2015, Damping properties of a beam with vacuum-packed granular damper, Journal of Sound and Vibration, 341, 74-85

2. Brown E., Rodenberg N., Amend J., et AL., 2010, Universal robotic gripper based on the jamming of granular material, Proceedings of the National Academy of Sciences of the United States of America, 107, 44, 18809-18814

3. Cates M.E., Wittmer J.P., Bouchaud J.P., Claudin P., 1998, Jamming, force chains and fragile matter, Physical Review Letters, 81, 1841-1844

4. Loeve A.J., Van de Ven O.S., Vogel J.G., Breedveld P., Dankelmann J., 2010, Vacuum packed particles as flexible endoscope guides with controllable rigidity, Granul Matter, 12, 6, 543-54

5. Luscombe M.D., Williams J.L., 2003, Comparison of a long spinal board and vacuum mattress for spinal immobilization, Emergency Medicine Journal, 20, 1, 476-478 
6. Majmudar T.S., Sperl M., Lundig S., Behringer R.P., 2007, Jamming transition in granular systems, Physical Reviews Letters, 98, 058001

7. Makowski M., KnAP L., 2014, Reduction of wheel force variations with magnetorheological devices, Journal of Vibration and Control, 20, 10, 1552-1564

8. Zalewski R., Pyrz M., 2013, Experimental study and modeling of polymer granular structures submitted to internal underpressure, Mechanics of Materials, 57, 75-85

Manuscript received June 10, 2014; accepted for print November 29, 2015 